\title{
Natural transverse vibration characteristics of jack-up riser and its applied research
}

\author{
Fengde Wang ${ }^{1, a}$, Wensheng Xiao ${ }^{1}$, Hongyan Wang ${ }^{2}$ Junguo Cui ${ }^{1}$, Jinsheng \\ Song $^{1}$, DingLi Wang ${ }^{1}$
}
${ }^{1}$ College of Mechanical and Electronic Engineering in China University of Petroleum, Qingdao 266580, China;

${ }^{2}$ College of Mechanical and Electronic Engineering in Qingdao University of Science \& Technology, Qingdao 266061, China.

âwangfengde110@163.com

Keywords: jack-up rig, riser, resonance, frequency equation

\begin{abstract}
In order to resolve the resonance problem of jack-up riser with drill string, the frequency equation of natural transverse vibration with compressive axial load and the length of the riser above the seabed as independent variable was derived in this paper. Then the frequency equation was solved by the Newton method, and the accuracy of the frequency calculation method without axial load was analyzed. In addition, a resonant rotating speed table of top-drive system (TDS) was made according to the relationship between rotating speed and frequency. The results show that: the method proposed in this paper is more accurate than the Rayleigh method; equation(22) is available when the length of the riser above the seabed is shorter than 30 meters while axial weight less than 90 tons ; there is a nonlinear relationship between the frequencies of the riser and the length of the riser above the seabed, and the frequencies decrease with the increasing length; under the general working conditions of jack-up , the relationship between the frequencies of the riser and axial compressive load is almost linear, and the frequencies decrease with the increasing axial compressive load; the resonant frequency range of the riser with drill string depends on the length of the riser above the seabed, and the resonant frequency range is extended by the increasing length.
\end{abstract}

\section{Introduction}

During the drill process of jack-up rig, one end of marine drill riser is fixed on the seabed and the other end is hinged to the jack-up platform [1, 2]. Since there is a BOP stack installed on the hinged end of the riser, which generates an axial compressive load to the riser. Therefore, the dynamic model of jack-up riser can be defined as a fixed-hinged uniform Euler-Bernoulli beam with axial compressive load. At present, the research of the jack-up drill riser is mainly concentrated in two aspects: natural transverse vibration characteristics of riser and its elastic stability [3, 4].

When jack-up rig was operated in the Bohai Sea, a phenomenon of violent vibration in the hinged end of the riser was discovered, which led to the failure of the top screw[4], in order to solve the above-mentioned problem, natural transverse vibration characteristics of riser was studied by the Rayleigh method in reference [4]. However, the Rayleigh method can only work out approximate results of the natural transverse vibration frequency, and the Rayleigh method also has a bigger error in calculating high-order frequencies [5]. The kinematic equation of the uniform beam with axial load was derived by F.J. Shaker [6], based on the kinematic equation, the axial load impact on natural frequency and mode of beam were researched. The free vibration characteristics analysis of a beam subjected to an axial tensile load with an attached in-span mass-spring-mass system was carried out by O. R. Barry [7]. The natural transverse vibration of a continuous beam with elastic supports subjected to axial load was researched with the Laplace Transform by Junqiang Li [8]. The post-buckling behavior of a slender beam with axial load in a circular tube was studied by M. G. Munteanu [9].The governing equation of transverse vibration of a free-free axially moving Timoshenko beam under an axial compressive load is established by Hong-yong CHEN [10], and the 
vibration characteristics of the beam are obtained by the analytical solution and Differential Quadrature Method (DQM).

Aiming at the problem proposed in reference [4], on the base of related research results and actual working conditions of jack-up, the frequency equation of natural transverse vibration with axial load and the length of riser above the seabed as independent variable was derived in this paper. The method proposed in this paper is more accurate than the Rayleigh method in computing frequency. In addition, the impact of axial compressive load and the length of the riser above the seabed were studied, on the base of that, a resonant rotation speed table of rig was made. The computing method of natural transverse vibration frequency of riser and the resonant rotating speed table can be used to guide the drill process of jack-up.

\section{Natural transverse vibration characteristics of the fixed-hinged beam with axial force}

Since the slenderness ratio of the riser is large enough, therefore the effects of shear deformation and rotary inertia can be neglected, and hence the Euler-Bernoulli beam theory is appropriate to be used in this study. Natural frequencies and vibration mode functions of the beam can be obtained through the free vibration analysis, the free transverse vibration equation of uniform beam with axial load is [6]:

$$
m \frac{\partial^{2} u(x, t)}{\partial t^{2}}+F \frac{\partial^{2} u(x, t)}{\partial x^{2}}+\frac{\partial^{2}}{\partial x^{2}}\left[E I \frac{\partial^{2} u(x, t)}{\partial x^{2}}\right]=0
$$

In Eq.(1), $t$ is the time, $x$ is the coordinate measured along the beam axis, $E$ is the modulus of elasticity, $I$ is the moment of inertia of the beam, $m$ is per unit length mass, $F$ is axial force, $u(\mathrm{x}, \mathrm{t})$ is the transverse deflection of the beam axis.

$$
u(x, t)=\phi(x) q(t)
$$

$\phi(x)$ is the mode of the vibration, which does not change with time; and $q(t)$ is the amplitude of the vibration .

By using variables separation method, Eq.(1) can be transferred to Eq.(3):

$$
E I \frac{\phi^{\prime \prime \prime}(x)}{\phi(x)}+F \frac{\phi^{\prime \prime}(x)}{\phi(x)}=-m \frac{\ddot{q}(t)}{q(t)}
$$

Where $\phi^{\prime \prime \prime}(x), \phi^{\prime \prime}(x)$ is the fourth-order and second-order differential of $x, \ddot{q}(t)$ is the second-order differential of $t$. Eq.(3) can be divided into two independent ordinary differential equations as follows:

$$
\begin{aligned}
& \ddot{q}(t)+\omega^{2} q(t)=0 \\
& E I \phi^{\prime \prime \prime}(x)+F \phi^{\prime \prime}(x)-m \omega^{2} \phi(x)=0
\end{aligned}
$$

Two symbols are introduced in this study, one is frequency coefficient $a^{4}$, and another one is axial force impact factor $p$.

$$
a^{4}=\frac{m \omega^{2}}{E I} ; \quad p=\frac{F}{E I} .
$$

Where $\omega$ is natural frequency of the beam.

By substituting $a^{4}$ and $p$ into Eq.(4-b), Eq.(4-b) can be transferred to Eq.(6):

$$
\phi^{\prime \prime \prime}(x)+p \phi^{\prime \prime}(x)-a^{4} \phi(x)=0
$$

On the base of the related research, the solution form of Eq.(6) can be defined as follows:

$$
\phi(x)=C e^{s x}
$$

Where, $C$ is a constant.

By substituting Eq.(7) into Eq.(6), Eq.(6) can be transferred to Eq.(8):

$$
\left(s^{4}+p s^{2}-a^{4}\right) C e^{s x}=0
$$

Roots of the characteristic equation of Eq. (8) can be obtained as follows: 


$$
S_{1,2}= \pm i \alpha ; \quad S_{3,4}= \pm \beta
$$

Where:

$$
\begin{gathered}
\alpha=\sqrt{\sqrt{a^{4}+\frac{p^{2}}{4}}+\frac{p}{2}} \\
\beta=\sqrt{\sqrt{a^{4}+\frac{p^{2}}{4}}-\frac{p}{2}}
\end{gathered}
$$

By substituting the solution into Eq.(7), the general solution of Eq.(4-b) can be obtained as follows:

$$
\phi(x)=C_{1} e^{i \alpha x}+C_{2} e^{-i \alpha x}+C_{3} e^{\beta x}+C_{4} e^{-\beta x}
$$

In Eq.(11), $C_{1} 、 C_{2} 、 C_{3} 、 C_{4}$ are constant. The exponential functions in above equation are substituted for trigonometric functions and hyperbolic functions, and then Eq.(11) can be transferred to Eq.(12) as follows:

$$
\phi(x)=A \sin (\alpha x)+B \cos (\alpha x)+C \sinh (\beta x)+D \cosh (\beta x)
$$

Where $A, B, C, D$ are constant.

The boundary conditions of the fixed end $(\mathrm{x}=0)$ are as follows:

$$
\begin{aligned}
& \phi(0)=0 \\
& \phi^{\prime}(0)=0
\end{aligned}
$$

Substitute Eq.(13) into Eq.(12), the relationship among coifficients in Eq.(12) can be obtained as follows:

$$
\begin{gathered}
B+D=0 \\
A \alpha+C \beta=0
\end{gathered}
$$

The boundary conditions of the hinged end $(\mathrm{x}=L)$ are as follows:

$$
\begin{aligned}
& \phi(L)=0 \\
& \phi^{\prime \prime}(L)=0
\end{aligned}
$$

Where, $L$ is the length of the riser above the seabed.

Eq.(16) can be obtained by substituting Eq.(15-a) into Eq.(12), as follows:

$$
A \sin (\alpha L)+B \cos (\alpha L)+C \sinh (\beta L)+D \cosh (\beta L)=0
$$

Eq.(17) can be obtained by substituting Eq.(15-b) into Eq.(12), as follows:

$$
-A \alpha^{2} \sin (\alpha L)-B \alpha^{2} \cos (\alpha L)+C \beta^{2} \sinh (\beta L)+D \beta^{2} \cosh (\beta L)=0
$$

The equation set of modal coefficient can be obtained by substituting Eq.(14) into Eq.(16)、(17):

$$
\begin{gathered}
{\left[\sinh (\beta L)-\frac{\beta}{\alpha} \sin (\alpha L)\right] C+[\cosh (\beta L)-\cos (\alpha L)] D=0} \\
{\left[\beta^{2} \sinh (\beta L)+\alpha \beta \sin (\alpha L)\right] C+\left[\beta^{2} \cosh (\beta L)+\alpha^{2} \cos (\alpha L)\right] D=0}
\end{gathered}
$$

In order to make the above equations have a nonzero solution, the value of coefficient determinant have to be zero, therefore the frequency equation of the beam can be obtained as follows:

$$
\sqrt{\beta^{2}+p} \tanh (\beta L)=\beta \tan \left(L \sqrt{\beta^{2}+p}\right)
$$

The parameters of $L$ and $p$ in Eq.(19) can be obtained by the working conditions of jack-up rig and the parameters of the riser. Because the closed-form expression of Eq.(19) can not be obtained at present, the parameter of $\beta$ is solved by the Newton method in this paper. On the base of that, by substituting the value of $\beta$ into the Eq.(10-b) and combining the Eq.(5), the natural frequencies of the riser can be obtained.

\section{Transverse vibration characteristic analysis of the riser under the working conditions}

According to the actual operating water depth of jack-up drilling platform, the regular range of the riser length above the seabed is $10 \sim 130 \mathrm{~m}$. A typical riser was analyzed in this paper, whose external diameter is $762.0 \mathrm{~mm}$, inner diameter is $711.2 \mathrm{~mm}$ and linear density is $461 \mathrm{~kg} / \mathrm{m}(\mathrm{E}=2.06 \mathrm{e} 11 \mathrm{~Pa})$. The 
axial force is expressed by impact factor $p$, whose range of values is $1 \times 10^{-4} \sim 11 \times 10^{-4} \mathrm{~m}^{-2}$. Within the scope of the above working conditions, the natural frequencies of the riser are computed by using equation (19)、(10-b)、(5-a) and (5-b). The results show that the minimum value of the sixth order of the natural frequency is $40.3268 \mathrm{rad} / \mathrm{s}$, which far more than the frequency range of the TDS. Therefore, only the first-order frequency to fifth-order frequency have to be calculated, which are shown in table 1 to table 5 .

On the base of frequencies data, the functional relationship between the fundamental frequency of the riser and the axial compressive load has been graphed by fig.1; and the relationship between the fundamental frequency of the riser and the length of riser above the seabed has been depicted in fig.2. As shown in fig.1, the relationship between the frequency of riser and the axial compressive load is almost linear under the general working conditions of jack-up rig, and the frequencies decrease with the increasing axial compressive load. As shown in fig.2, there is a nonlinear relationship between the frequencies of the riser and the length of the riser above the seabed, and the frequencies decrease with the increasing length.

Table 1 Fundamental frequency of the riser

\begin{tabular}{cccccccc}
\hline$\omega_{1}$ & $\mathrm{~L}=10$ & $\mathrm{~L}=30$ & $\mathrm{~L}=50$ & $\mathrm{~L}=70$ & $\mathrm{~L}=90$ & $\mathrm{~L}=110$ & $\mathrm{~L}=130$ \\
\hline $\mathrm{p}=1 \mathrm{e}-4$ & 206.3464 & 22.8701 & 8.2005 & 4.1400 & 2.5036 & 1.6568 & 1.1739 \\
$\mathrm{p}=3 \mathrm{e}-4$ & 206.1650 & 22.7593 & 8.0837 & 4.0653 & 2.3959 & 1.5481 & 1.0579 \\
$\mathrm{p}=5 \mathrm{e}-4$ & 205.8789 & 22.6489 & 8.0089 & 3.9491 & 2.2813 & 1.4438 & 0.9352 \\
$\mathrm{p}=7 \mathrm{e}-4$ & 205.6979 & 22.5390 & 7.8944 & 3.8355 & 2.1601 & 1.3043 & 0.7928 \\
$\mathrm{p}=9 \mathrm{e}-4$ & 205.5171 & 22.4981 & 7.8010 & 3.7374 & 2.0517 & 1.1624 & 0.6144 \\
$\mathrm{p}=11 \mathrm{e}-4$ & 205.3365 & 22.3888 & 7.6694 & 3.6146 & 1.9173 & 1.0030 & 0.3505 \\
\hline
\end{tabular}

Table 2 Second-order frequency of the riser

\begin{tabular}{cccccccc}
\hline$\omega_{2}$ & $\mathrm{~L}=10$ & $\mathrm{~L}=30$ & $\mathrm{~L}=50$ & $\mathrm{~L}=70$ & $\mathrm{~L}=90$ & $\mathrm{~L}=110$ & $\mathrm{~L}=130$ \\
\hline $\mathrm{p}=1 \mathrm{e}-4$ & 668.3369 & 74.3400 & 26.6465 & 13.5970 & 8.1797 & 5.4743 & 3.8929 \\
$\mathrm{p}=3 \mathrm{e}-4$ & 667.7146 & 74.0330 & 26.5538 & 13.4617 & 8.0837 & 5.3360 & 3.7826 \\
$\mathrm{p}=5 \mathrm{e}-4$ & 667.2815 & 73.8524 & 26.4614 & 13.3806 & 7.9481 & 5.2333 & 3.6614 \\
$\mathrm{p}=7 \mathrm{e}-4$ & 667.0375 & 73.6721 & 26.2949 & 13.2473 & 7.8147 & 5.1004 & 3.5302 \\
$\mathrm{p}=9 \mathrm{e}-4$ & 666.7935 & 73.5548 & 26.1293 & 13.1153 & 7.7223 & 4.9703 & 3.4025 \\
$\mathrm{p}=11 \mathrm{e}-4$ & 666.5496 & 73.4376 & 26.1120 & 13.0102 & 7.6114 & 4.8577 & 3.2655 \\
\hline \multicolumn{7}{c}{ Table 3 Third-order frequency of the riser } \\
\hline$\omega_{3}$ & $\mathrm{~L}=10$ & $\mathrm{~L}=30$ & $\mathrm{~L}=50$ & $\mathrm{~L}=70$ & $\mathrm{~L}=90$ & $\mathrm{~L}=110$ & $\mathrm{~L}=130$ \\
\hline $\mathrm{p}=1 \mathrm{e}-4$ & 1394.6 & 156.4411 & 55.7051 & 28.4091 & 17.1684 & 11.4565 & 8.1797 \\
$\mathrm{p}=3 \mathrm{e}-4$ & 1393.9 & 154.7511 & 55.5660 & 28.2703 & 16.9999 & 11.3187 & 8.0837 \\
$\mathrm{p}=5 \mathrm{e}-4$ & 1393.7 & 154.7028 & 55.4816 & 28.1322 & 16.8926 & 11.2067 & 7.9481 \\
$\mathrm{p}=7 \mathrm{e}-4$ & 1392.5 & 154.4729 & 55.3432 & 27.9947 & 16.7563 & 11.0718 & 7.8147 \\
$\mathrm{p}=9 \mathrm{e}-4$ & 1392.1 & 154.3340 & 55.2050 & 27.8961 & 16.6797 & 10.9620 & 7.6832 \\
$\mathrm{p}=11 \mathrm{e}-4$ & 1391.7 & 154.2859 & 55.0672 & 27.7597 & 16.5450 & 10.8297 & 7.5728 \\
\hline
\end{tabular}

Table 4 Fourth-order frequency of the riser

\begin{tabular}{cccccccc}
\hline$\omega_{4}$ & $\mathrm{~L}=10$ & $\mathrm{~L}=30$ & $\mathrm{~L}=50$ & $\mathrm{~L}=70$ & $\mathrm{~L}=90$ & $\mathrm{~L}=110$ & $\mathrm{~L}=130$ \\
\hline $\mathrm{p}=1 \mathrm{e}-4$ & 2384.6 & 264.6962 & 95.3048 & 48.5339 & 29.3512 & 19.6409 & 14.0309 \\
$\mathrm{p}=3 \mathrm{e}-4$ & 2384.0 & 264.5919 & 95.1531 & 48.4638 & 29.2473 & 19.5157 & 13.8913 \\
$\mathrm{p}=5 \mathrm{e}-4$ & 2383.1 & 264.4877 & 95.0016 & 48.3431 & 29.1438 & 19.3594 & 13.7800 \\
$\mathrm{p}=7 \mathrm{e}-4$ & 2382.5 & 264.3835 & 94.9214 & 48.2226 & 29.0015 & 19.2676 & 13.6694 \\
$\mathrm{p}=9 \mathrm{e}-4$ & 2381.9 & 264.2794 & 94.7702 & 48.1025 & 28.8210 & 19.1447 & 13.5333 \\
$\mathrm{p}=11 \mathrm{e}-4$ & 2381.3 & 264.1753 & 94.6193 & 47.9324 & 28.7577 & 19.0225 & 13.4245 \\
\hline
\end{tabular}


Table 5 Fifth-order frequency of the riser

\begin{tabular}{cccccccc}
\hline$\omega_{5}$ & $\mathrm{~L}=10$ & $\mathrm{~L}=30$ & $\mathrm{~L}=50$ & $\mathrm{~L}=70$ & $\mathrm{~L}=90$ & $\mathrm{~L}=110$ & $\mathrm{~L}=130$ \\
\hline $\mathrm{p}=1 \mathrm{e}-4$ & 3637.2 & 404.1983 & 145.3954 & 74.0882 & 44.8397 & 29.9878 & 21.4609 \\
$\mathrm{p}=3 \mathrm{e}-4$ & 3636.9 & 404.0379 & 145.2646 & 74.0330 & 44.6799 & 29.8814 & 21.3576 \\
$\mathrm{p}=5 \mathrm{e}-4$ & 3636.6 & 403.8777 & 145.1340 & 73.9151 & 44.5693 & 29.7356 & 21.2214 \\
$\mathrm{p}=7 \mathrm{e}-4$ & 3635.9 & 403.7175 & 145.0034 & 73.7973 & 44.4589 & 29.6696 & 21.0527 \\
$\mathrm{p}=9 \mathrm{e}-4$ & 3635.6 & 403.5574 & 144.8730 & 73.6798 & 44.3488 & 29.4855 & 20.9514 \\
$\mathrm{p}=11 \mathrm{e}-4$ & 3635.3 & 403.3973 & 144.7427 & 73.5000 & 44.1907 & 29.3416 & 20.8504 \\
\hline
\end{tabular}

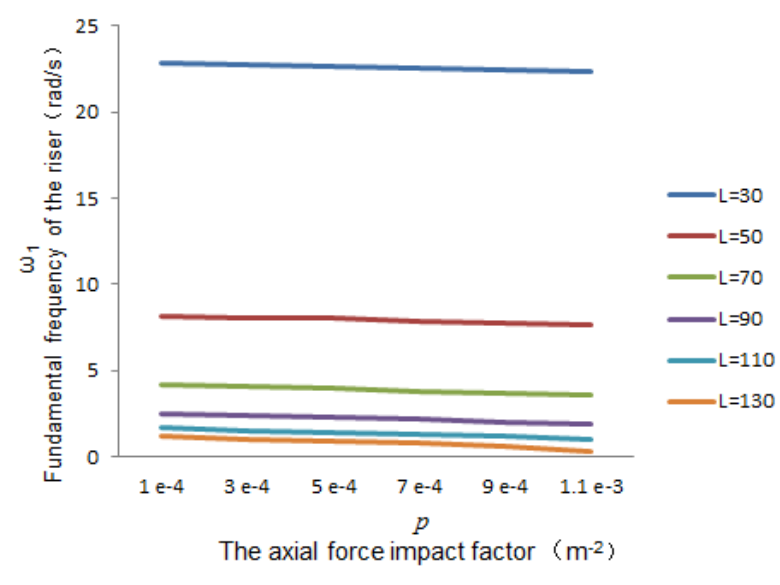

Fig.1 The relationship between $\omega_{1}$ and $p$

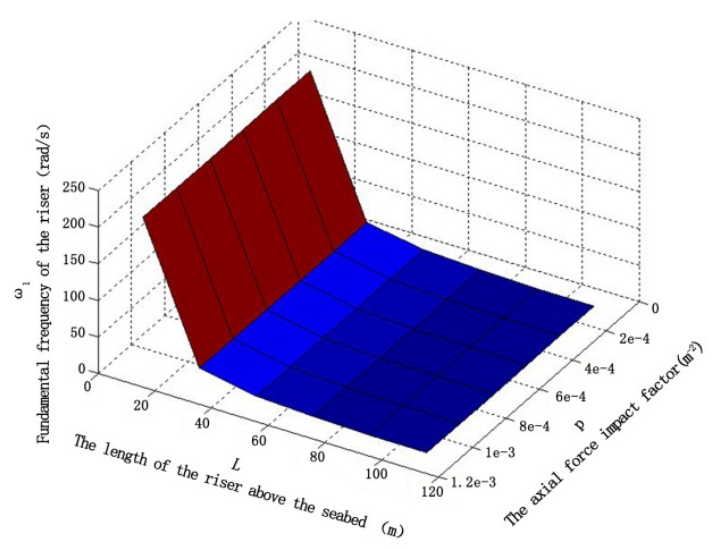

Fig. 2 The relationship between $\omega_{1}$ and $L, p$

When taking no account of the axial load, the frequency equation of the fixed-hinged beam is:

$$
\tanh (a L)=\tan (a L)
$$

This transcendental equation can be solved as follows:

$$
a_{n} L \approx\left(n+\frac{1}{4}\right) \pi \quad(n=1,2,3 \ldots)
$$

By substituting the Eq. (5-a) into the above equation, natural frequencies of beam without axial load can be obtained as follows:

$$
\omega_{n}=\frac{\left(n+\frac{1}{4}\right)^{2} \pi^{2}}{L^{2}} \sqrt{\frac{E I}{m}} \quad(n=1,2,3, \ldots)
$$

In order to evaluate the accuracy of the results by Eq. (22), a comparative analysis of results was carried out, and a conclusion can be obtained as follows: when the length of riser above the seabed is shorter than 30m while axial weight less than 90 tons, the error of the results by Eq. (22) are less than 2.5\%, therefore Eq. (22) is suitable for calculating the natural frequency of the riser in these working conditions.

\section{Resonant Rotation Speed Table}

A typical rotating speed range of the TDS used in offshore drilling is up to $273 \mathrm{r} / \mathrm{min}$, the frequency range of the TDS can be obtained by equation (23):

$$
\omega=\frac{n \pi}{30}
$$

and the frequency range of the TDS is $0 \sim 28.5885 \mathrm{rad} / \mathrm{s}$, on the base of that, a resonant rotating speed table under different working conditions can be made (as shown in table 6). When a jack-up rig operated under a certain working condition, the engineers can avoid resonant rotating speed by this table. In addition, the results in table 6 show that the orders of the marine riser's resonant frequency is 
mainly decided by the length of the riser above the seabed, which increases as the increasing length of riser above the seabed.

Table 6 Resonant rotating speed of jack-up rig under different working conditions

\begin{tabular}{|c|c|c|c|c|c|c|c|}
\hline $\begin{array}{l}\text { The length of } \\
\text { the riser above } \\
\text { the seabed }\end{array}$ & $\begin{array}{c}\text { Orders of } \\
\text { resonant } \\
\text { rotation speed }\end{array}$ & $p=1 \mathrm{e}-4$ & $p=3 e-4$ & $p=5 \mathrm{e}-4$ & $p=7 \mathrm{e}-4$ & $p=9 \mathrm{e}-4$ & $p=11 \mathrm{e}-4$ \\
\hline$L=30$ & $n_{30}^{1}$ & 218.3934 & 217.3353 & 216.2811 & 215.2316 & 214.8410 & 213.7973 \\
\hline \multirow{3}{*}{$L=50$} & $n_{50}^{1}$ & 78.3090 & 77.1936 & 76.4794 & 75.3860 & 74.4941 & 73.2374 \\
\hline & $n_{50}^{2}$ & 254.4553 & 253.5701 & 252.6878 & 251.0978 & 249.5164 & 249.3512 \\
\hline & $n_{70}^{1}$ & 39.5341 & 38.8208 & 37.7111 & 36.6263 & 35.6895 & 34.5169 \\
\hline \multirow[t]{3}{*}{$L=70$} & $n_{70}^{2}$ & 129.8418 & 128.5498 & 127.7753 & 126.5024 & 125.2419 & 124.2383 \\
\hline & $n_{70}^{3}$ & 271.2869 & 269.9615 & 268.6427 & 267.3297 & 266.3881 & 265.0856 \\
\hline & $n_{90}^{1}$ & 23.9076 & 22.8792 & 21.7848 & 20.6274 & 19.5923 & 18.3089 \\
\hline \multirow[t]{3}{*}{$L=90$} & $n_{90}^{2}$ & 78.1104 & 77.1936 & 75.8988 & 74.6249 & 73.7425 & 72.6835 \\
\hline & $n_{90}^{3}$ & 163.9461 & 162.3371 & 161.3124 & 160.0109 & 159.2794 & 157.9931 \\
\hline & $n_{110}^{1}$ & 15.8213 & 14.7833 & 13.7873 & 12.4551 & 11.1001 & 9.5779 \\
\hline \multirow{5}{*}{$L=110$} & $n_{110}^{2}$ & 52.9728 & 50.9550 & 49.9743 & 48.7052 & 47.4629 & 46.3876 \\
\hline & $n_{110}^{3}$ & 109.4015 & 108.0856 & 107.0161 & 105.7279 & 104.6794 & 103.4160 \\
\hline & $n_{110}^{4}$ & 187.5568 & 186.3612 & 184.8687 & 183.9920 & 182.8184 & 181.6515 \\
\hline & $n_{130}^{1}$ & 11.2099 & 10.1022 & 8.9305 & 7.5707 & 5.8671 & 3.3470 \\
\hline & $n_{130}^{2}$ & 37.1745 & 36.1212 & 34.9638 & 33.7109 & 32.4915 & 31.1832 \\
\hline \multirow[t]{3}{*}{$L=130$} & $n_{130}^{3}$ & 78.1104 & 77.1936 & 75.8988 & 74.6249 & 73.3692 & 72.3149 \\
\hline & $n_{130}^{4}$ & 133.9852 & 132.6521 & 131.5893 & 130.5332 & 129.2335 & 128.1945 \\
\hline & $n_{130}^{5}$ & 204.9365 & 203.9501 & 202.6494 & 201.0385 & 200.0711 & 199.1067 \\
\hline
\end{tabular}

Note: $n_{L}^{b}$, where $b$ is the order of the rotating speed.

\section{Summary}

The frequency equation of jack-up riser's natural transverse vibration was derived in this paper, which takes axial compressive load and the length of the riser above the seabed as independent variable. Then the frequency equation was solved by the newton method. On the base of that, natural transverse vibration characteristics of jack-up riser were analyzed in detail and a resonant rotating speed table was made, which can be used to guide the drill process of jack-up rig. From this study, the following conclusions are reached:

1. The frequency calculation method proposed in this paper is more accurate than the Rayleigh method; if the length of the riser above the seabed is shorter than 30 meters while axial weight less than 90 tons, Eq.(22) is accurate enough to compute the frequencies.

2. There is a nonlinear relationship between the frequency of riser and the length of the riser above the seabed, and the frequencies decrease with the increasing length.

3. Under the general working conditions of jack-up, the relationship between the frequencies of the 
riser and axial compressive load is almost linear, and the frequencies decrease with the increasing axial compressive load.

4. The resonant frequency range of the riser with drill string depends on the length of the riser above the seabed, and the resonant frequency range is extended by the increasing length.

\section{References}

[1] Jin Yang, Shujie Liu, Jianliang Zhou, et al. Strength and security calculation on riser with the effect of wind, wave and current. China Offshore Oil and Gas, 2006, 18(3): 198-200.

[2] Qinfeng Hu. Wellhead stability analysis of offshore self-elevating exploratory well in drilling platform. Oil Drilling \& Production Technology, 2007, 29(6): 10-12.

[3] Wei Jiang. Study on critical load and elastic stability of drilling riser. China Offshore Oil and Gas, 2006, 18(6): 403-407.

[4] Wei Jiang. Lateral vibration performance of drilling riser and its impact to drilling operation. China Offshore Oil and Gas, 2007, 19(6): 394-397.

[5] Jingbo Liu, Xiuli Du. Structural Dynamics. Beijing: China Machine Press, 2014: 207.

[6] F.J. Shaker. Effect of axial load on mode shapes and frequencies of beams. Lewis Research Center Report NASA-TN-8109, December 1975.

[7] O. R. Barry, Y Zhu, J.W. Zu, et al. Free vibration analysis of a beam under axial load carrying a mass-spring-mass. ASME 2012 International Design Engineering Technical Conferences and Computers and Information in Engineering Conference. 2012: 791-796.

[8] Junqiang Li, Fang Tong. The natural transverse vibration of a continuous beam with elastic supports subjected to axial load. Journal of XI'AN Shiyou University(Natural Science Edition), 1999(4): 70-72.

[9] M. G. Munteanu, Baracco. Post-buckling behavior of a slender beam in a circular tube under axial load. Computational Methods \& Experimental Measurements, 2007 XIII (46):547-556.

[10] H Y Chen, H B Chen. Vibration characteristics of free-free moving beam under axial compressive loads. Engineering Mechanics, 2015, 32(3): 233-240.

[11] S. Timoshenko. Vibration problems in engineering. Hu R L, tran. Beijing: People's Railway Press, 1978: 291.

[12] Mosheng Liao. Offshore oil drilling engineering technology and equipment. Beijing: China Petrochemical Press, 2010: 148. 